\title{
Auditorías de seguridad vial de carreteras en operación
}

\section{In-Service Road Safety Audits}

\author{
A. Mendoza-Díaz \\ Instituto Mexicano del Transporte, México. \\ E-mail:Alberto.Mendoza@imt.mx \\ E. Abarca-Pérez \\ Instituto Mexicano del Transporte, México. \\ E-mail:Emilio.Abarca@imt.mx \\ A.G. Centeno-Saad \\ Instituto Mexicano del Transporte, México. \\ E-mail:acenteno@imt.mx
}

(Recibido: mayo de 2007; aceptado: abril de 2008)

\section{Resumen}

Una auditoría de seguridad vial es un análisis formal que pretende garantizar que un camino existente o futuro cumpla con criterios óptimos de seguridad, llevado a cabo por un equipo de expertos, cuyos miembros son independientes del proyecto del camino. Puede realizarse en una, varias o todas las etapas del proyecto (planeación, proyecto, construcción, antes de abrir el camino al tránsito y operación). El desarrollo e implantación de un proceso de auditorías de seguridad en carreteras es una de las estrategias que se han aplicado en México en los últimos años para reducir la accidentalidad y sus consecuencias asociadas. El objetivo de este trabajo es presentar los procedimientos que se han desarrollado y aplicado en México, así como los beneficios y problemas que se han encontrado. Se ilustra también la aplicación de esos procedimientos, a un caso específico. Se hace énfasis en la auditoría de carreteras en operación, que es el tipo que mayor aplicación y desarrollo ha tenido en México.

Descriptores: Auditoría, seguridad vial, carretera, proyecto, inspección, normatividad.

\begin{abstract}
A road safety audit is a formal analysis that seeks to guarantee that an existing or future road fulfills optimal safety criteria, conducted by a team of experts whose members are independent of the road project. It can be carried out in one, several or all of the stages of the project (planning, design, construction, before opening the road to traffic, and operation). The development and implementation of a road safety audit process is one of the strategies that have been applied in Mexico in recent years to reduce road accidents and their associated consequences. The objective of this work is to present the procedures that have been developed and applied in Mexico, as well as the benefits and problems that have been encountered. The application of those procedures to a specific case is also shown. Emphasis is made in the audit of highways in the operation stage, which is the type that greater application and development has had in Mexico.
\end{abstract}

Keywords: Audit, safety, road, project, inspection, regulation. 
DOI: http://dx.doi.org/10.22201/fi.25940732e.2009.10n2.013

Auditorías de seguridad vial de carreteras en operación

\section{Introducción}

Mejorar sustancialmente la seguridad vial en carreteras continúa siendo motivo de gran preocupación y ocupación en todos los países.

Una estrategia que se ha utilizado recientemente en México durante los últimos años para tratar de reducir la accidentalidad y sus consecuencias asociadas, ha sido la aplicación de medidas correctivas. Sin embargo, este tipo de medidas, desde el punto de vista de la seguridad vial, representan una solución a un problema manifiesto, causa de un número significativo de accidentes, lesionados y muertos.

En años recientes, se ha buscado complementar la estrategia anterior con un enfoque que, en lugar de ser una solución a un problema manifiesto, contribuya a prevenirlo mediante un análisis de riesgos. Uno de los principales esfuerzos dirigidos a este fin, ha sido el desarrollo e implantación de un proceso de auditorías de seguridad en carreteras.

El objetivo de este trabajo es presentar los procedimientos que se han desarrollado y aplicado en México, así como los beneficios y problemas que se han encontrado. Se ilustra la aplicación de esos procedimientos, a un caso específico.

Se enfatiza en la auditoría de carreteras en operación, que es el tipo que mayor aplicación y desarrollo que se ha tenido en México. Este tipo de auditorías son preventivas, en cuanto a corregir errores antes de que ocurran los accidentes. En este trabajo, por la palabra auditor, deberá entenderse que, más que a una persona en particular, se hace referencia a un equipo de dos o más personas encargados de la auditoría.

\section{Procedimientos desarrollados y aplicados en México}

Se ha partido de la definición de que una Auditoría de Seguridad Carretera es un proceso que pretende garantizar que los caminos, desde su etapa de planeación, se diseñen con criterios óptimos de seguridad para todos los usuarios, verificando que se mantengan dichos criterios durante las etapas de proyecto, construcción y puesta en servicio de la misma (Austroroads, 2004).

Uno de los aspectos más relevantes dentro del proceso de aplicación de una Auditoría de Seguridad Carretera es determinar el momento en que ésta se llevará a cabo. Una auditoría se puede llevar a cabo en una, varias o todas las etapas de un proyecto (planeación, proyecto, construcción, antes de abrir el camino al tránsito y operación).
En México, la mayoría de los avances y aplicaciones se han generado en torno a los caminos en la etapa de operación, a través de un programa anual dirigido a identificar todos aquellos elementos que representen un riesgo potencial para la seguridad de los usuarios. El énfasis se ha puesto en mejorar la condición de los 14 mil kilómetros de corredores troncales principales, los cuales comunican los sitios más importantes del país entre sí (capitales de los estados, ciudades, puertos y fronteras) y con el exterior. A continuación, se describen los pasos que se han seguido en México en la realización de auditorías de caminos en operación.

\section{Solicitud de la auditoría}

En el caso de carreteras en operación, la autoridad responsable de la operación de la misma es la encargada de solicitar la auditoría.

Una vez realizada la solicitud, la autoridad responsable de la operación de la carretera establece en los términos de referencia, los alcances de la auditoría, es decir, la etapa o etapas en las que se llevará a cabo, además de establecer también la forma en que se efectuará el trabajo, los datos básicos de la carretera que serán entregados y el contenido del reporte que deberá entregar el auditor al finalizar el trabajo.

\section{Recopilación de información necesaria}

La autoridad responsable de la operación de la carretera también proporciona al auditor los datos de entrada necesarios, que una vez entregados, son revisados por el mismo auditor, quien coteja que la información contenga los siguientes datos básicos:

a) El nombre de la carretera y los tramos a auditar (según su kilómetro de inicio y kilómetro final).

b) El tipo de carretera, de acuerdo con el Reglamento de Pesos y Dimensiones (SCT, 2000).

c) El croquis o plano de localización de la carretera.

d) Volúmenes de tránsito y su composición vehicular.

e) Información sobre las condiciones del entorno vial, incluyendo los servicios, el clima, la fauna, la flora, la topografía, etc.

f) Una copia de toda la normatividad aplicable.

g) Los planos donde se muestra el alineamiento horizontal, vertical y los detalles de las secciones del camino.

h) Un historial de accidentes de la carretera, en donde se incluyen los periodos, los tipos, la distribución temporal y la frecuencia. 
DOI: http://dx.doi.org/10.22201/fi.25940732e.2009.10n2.013

Revisión y evaluación de la información

Se revisa que la información obtenida esté ordenada y que tenga una fácil interpretación, permitiendo tener una idea clara de lo que se va a revisar en la inspección de campo, lo cual sirve además para dar una valoración sobre ciertos aspectos de la carretera antes de realizar la inspección.

Inspección de campo

Se lleva a cabo una vez que se revisó la información y se constató que se cuenta con los datos suficientes y las anotaciones necesarias para ser tomadas en cuenta durante la inspección de campo.

Esta inspección consiste en llevar a cabo el levantamiento y registro en campo de todos aquellos elementos de la carretera que pueden representar un riesgo para la seguridad de los usuarios, tales como la falta, deterioro o deficiencias del señalamiento horizontal y vertical, las deficiencias en las características físicas del pavimento, la falta de los elementos de protección y seguridad, todas aquellas características geométricas y operacionales del camino que no cumplen las especificaciones y normas, así como aquéllas que aún cumpliéndolas, el auditor considera que son elementos o condiciones que propician situaciones potenciales de peligro para la seguridad de los usuarios de la carretera. Este trabajo se realiza utilizando una serie de formatos, los cuales ayudan a realizar el levantamiento y el registro de datos en el campo.

En México se han utilizado los siguientes cuatro formatos: uno para el registro de las características físicas y geométricas de la carretera, otro para el registro del señalamiento vertical, otro más para el registro del señalamiento horizontal y el último para el registro de otros dispositivos, de los cuales ninguno cumple con la normatividad.

La figura 1 muestra el formato para el registro del señalamiento vertical que no cumple con la normatividad. En este caso, en la parte superior del formato se registran:

1. El nombre de la carretera auditada.

2. El nombre del tramo auditado.

3. La clasificación de la carretera de acuerdo con el Reglamento de Pesos y Dimensiones de los Vehículos de Carga.

4. La longitud del tramo auditado.

5. La fecha.

6. La hora de cuándo se realiza la inspección.

7. El nombre de la persona que la realiza.

En la parte inferior se van registrando, para cada señal vertical que no cumple con la normatividad:

8. Su cadenamiento (en kilómetros + metros).

9. Su clave de acuerdo con el Manual de Dispositivos para el Control del Tránsito en Calles y Carreteras.

10. El nombre y descripción de la señal.

11.El sentido de circulación al que sirve la misma, colocándose posteriormente una " $\mathrm{X}$ " en las columnas.

12. De los conceptos para los que la señal no cumple con lo normado de acuerdo con la clasificación de la carretera en el Reglamento de Pesos y Dimensiones (SCT, 2000). Asimismo se registran las señales que se requieren.

13) Instalar.

SEÑALAMIENTO VERTICAL DE CARRETERAS DE DOS, CUATRO O MÁS CARRILES QUE NO CUMPLEN CON LA NORMATIVIDAD

\begin{tabular}{|c|c|c|c|c|c|c|c|c|c|c|c|c|c|}
\hline $\begin{array}{l}\text { CARRETERA: } \\
\text { LONGITUD KM: }\end{array}$ & $(1)$ & & & $\begin{array}{l}\text { TRAMO: } \\
\text { FECHA: }\end{array}$ & (2) & & HORA: & (6) & & $\begin{array}{l}\text { TIPO DE } \\
\text { ING. RES }\end{array}$ & $\begin{array}{l}\text { CAMINO: } \\
\text { PONSABLE: }\end{array}$ & (3) & \\
\hline & & & & & & FORMATO & & & & & & & \\
\hline $\begin{array}{c}\text { LOCALIZACIÓN } \\
(\mathrm{KM}) \\
\end{array}$ & $\begin{array}{l}\text { CLAVE } \\
\text { SEÑAL }\end{array}$ & $\begin{array}{c}\text { NOMBRE Y/O } \\
\text { DESCRIPCIÓN DE LA } \\
\text { SEÑAL } \\
\end{array}$ & $\begin{array}{l}\text { SENTIDO DE } \\
\text { CIRCULACIÓN }\end{array}$ & $\begin{array}{l}\text { UBICACIÓN } \\
\text { LONG. }\end{array}$ & $\begin{array}{l}\text { UBICACIÓN } \\
\text { LATERAL }\end{array}$ & $\begin{array}{c}\text { DIMENSIÓN } \\
\text { DE LA } \\
\text { SEÑAL } \\
\end{array}$ & $\begin{array}{c}\text { NO CUMPLF } \\
\text { CONLOS } \\
\text { COLORES } \\
\end{array}$ & $\begin{array}{l}\text { NO CUMPLE } \\
\text { CON EL } \\
\text { DISEÑO } \\
\end{array}$ & $\begin{array}{c}\text { NO TIENE } \\
\text { REFLEJANTE }\end{array}$ & $\begin{array}{l}\text { ESTADO } \\
\text { FISICO }\end{array}$ & $\begin{array}{c}\text { SE } \\
\text { REQUIERE }\end{array}$ & RETIRAR & OBSERVACIONES \\
\hline (8) & (9) & (10) & (11) & (12) & (12) & (12) & (12) & (12) & (12) & (12) & (13) & (14) & (15) \\
\hline & & & & & & & & & & & & & \\
\hline & & & & & & & & & & & & & \\
\hline & & & & & & & & & & & & & \\
\hline & & & & & & & & & & & & & \\
\hline & & & & & & & & & & & & & \\
\hline & & & & & & & & & & & & & \\
\hline & & & & & & & & & & & & & \\
\hline & & & & & & & & & & & & & \\
\hline & & & & & & & & & & & & & \\
\hline
\end{tabular}

Figura 1. Formato que se utiliza para el registro del señalamiento vertical que no cumple con la normatividad 
DOI: http://dx.doi.org/10.22201/fi.25940732e.2009.10n2.013

Auditorías de seguridad vial de carreteras en operación

14. Retirar.

15. Las observaciones que se estimen pertinentes.

Los recorridos para las inspecciones de campo son tanto diurnos como nocturnos. Las anomalías detectadas y registradas en los formatos son fotografiadas, generándose con ello el testimonio visual del trabajo de campo.

Una vez terminada la inspección, el auditor lleva a cabo el trabajo de gabinete, donde procesa y analiza toda la información registrada en los formatos de campo para diagnosticar la situación que prevalece en los tramos de estudio.

Con los resultados del análisis y el diagnóstico, determina y señala los principales elementos que representan un riesgo en el camino y proponen en forma enunciativa las posibles soluciones para cada elemento analizado.

Reporte de la auditoría

Los resultados de la aplicación de la auditoría son comunicados a través de un reporte escrito, el cual incluye las recomendaciones de seguridad o acciones correctivas sobre los aspectos que involucran peligros innecesarios o irrazonables para los usuarios de la carretera. En el reporte no se incluyen los aspectos positivos de la carretera.

Las recomendaciones establecidas en el reporte se limitan a indicar la posible solución y no a desarrollar ésta a detalle.

\section{Ejemplo de aplicación}

El Instituto Mexicano del Transporte (IMT) realizó la auditoría de seguridad de la autopista de cuota México-Puebla, la cual tiene una longitud total de 110 kilómetros y un TDPA de alrededor de 30,000 vehículos.

La composición del tránsito en esta carretera incluye $20 \%$ de vehículos de carga, de los cuales $5 \%$ son tractocamiones.

En la dirección de México hacia Puebla, entre el kilómetro 0 y el 56 , esta carretera asciende con pendientes de entre el 4 y el $5 \%$, descendiendo con pendientes similares del kilómetro 56 hasta el 95.

Por su cercanía a la Ciudad de México, esta carretera se encuentra a más de 2 mil metros sobre el nivel de mar, por lo cual en buena parte del año (otoño e invierno) su superficie se encuentra mojada o con hielo, siendo también frecuente la presencia de niebla en el camino, particularmente durante las madrugadas.
El trazo de esta autopista es sinuoso, habiéndose detectado en ella gran incidencia de accidentes, particularmente en los tramos en descenso (del kilómetro 56 hasta el 95 en la dirección de México hacia Puebla y del 56 al 0 en la dirección de Puebla hacia México).

Para los dos tramos anteriores, se siguieron los pasos del procedimiento de auditoría antes descrito.

A manera de ejemplo, la figura 2 muestra un segmento de alta peligrosidad del tramo en descenso de México hacia Puebla, sentido en que el kilometraje crece. El segmento consiste en dos curvas continuas opuestas de grados 4.5 y 4 consecutivamente. La velocidad de proyecto de este segmento es de 100 kilómetro por hora y el límite máximo establecido de 90 kilómetros por hora. La carretera tiene dos carriles de $3.50 \mathrm{~m}$ en tangente, con acotamientos interno y externo de 0.50 y $1 \mathrm{~m}$, respectivamente. Ambas curvas cuentan con las ampliaciones, sobreelevaciones y transiciones requeridas por las Normas de Proyecto Geométrico de Carreteras (SCT, 1984).

La parte superior de la figura 2 ilustra la geometría y el señalamiento registrados para el segmento durante las inspecciones de campo.

Como resultado de la auditoría, se detectaron las siguientes anomalías:

a) Ambas curvas tienen grados mayores que el máximo correspondiente a sus velocidades de acuerdo con las normas de proyecto geométrico de carreteras (3.5 grados).

b) Para las condiciones del segmento, tanto el ancho de carril como el ancho de los acotamientos interno y externo son menores a los mínimos especificados por las normas de proyecto geométrico de carreteras (3.65, 1 y $2.5 \mathrm{~m}$, respectivamente).

c) Dado que los camiones de carga al circular por el carril de la derecha descienden a muy bajas velocidades (de manera similar a como lo hacen en el ascenso), se detectó que representan un serio riesgo para los autobuses y los vehículos ligeros que descienden a mucha mayor velocidad (a una velocidad promedio de más de 30 kilómetros por hora que la de los camiones de carga).

d)También se detectó la necesidad de mejorar el señalamiento vertical y horizontal, haciéndolo más claro y conspicuo. 

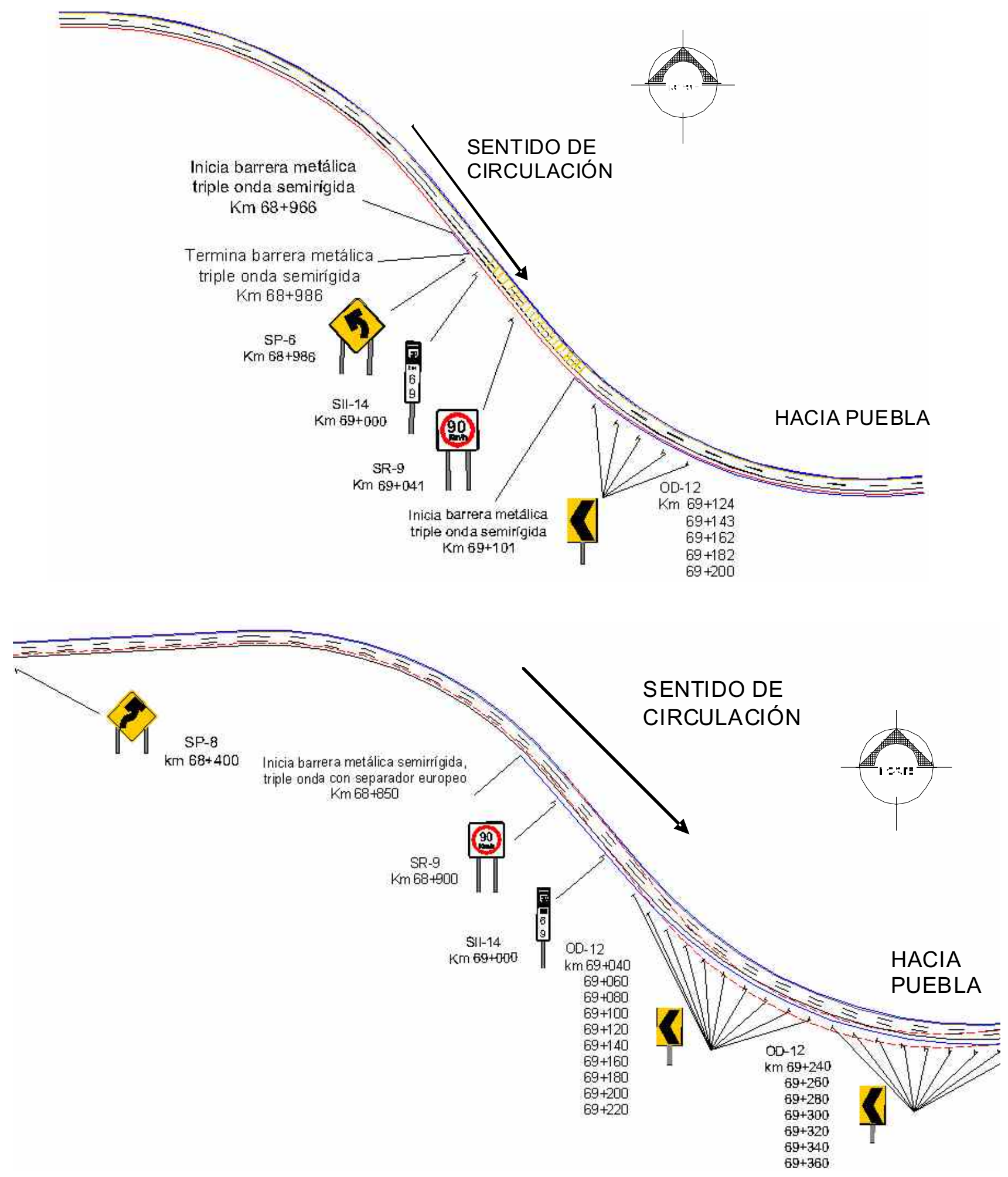

Figura 2. Segmento de alta peligrosidad del tramo en descenso de México hacia Puebla 
DOI: http://dx.doi.org/10.22201/fi.25940732e.2009.10n2.013

En el reporte de la auditoría se recomendó modernizar el segmento mediante la realización de las siguientes acciones:

a) Modificar las curvas, derecha e izquierda, disminuyendo el grado de curvatura a $3^{\circ}$, con carriles de $4 \mathrm{~m}$ de ancho al centro de la curva, aumentando la longitud de la misma y proporcionando acotamientos interno y externo de $1.00 \mathrm{~m}$ y $2.50 \mathrm{~m}$, respectivamente; $y$

b) Ampliar la sección transversal de 2 a 3 carriles de circulación, proporcionando un ancho mínimo de $3.65 \mathrm{~m}$ por carril en tangente. Esta ampliación deberá realizarse hacia el interior de la curva, incrementándose la visibilidad en el sitio y mejorando el trazo en el cuerpo "B". La localización definitiva del trazo será en función del estudio específico de proyecto geométrico que se realice para este caso.

c) Se recomendó también adicionar las obras de drenaje requeridas, de acuerdo al nuevo alineamiento, en función del estudio hidrológico detallado que deberá realizarse para este sitio.

La parte inferior de la figura 2 muestra los conceptos generales que deberán cubrir las modernizaciones mencionadas en cuanto al trazo y al señalamiento correspondiente. En esta parte, el trazo punteado en color rojo corresponde a la situación actual, en tanto que el trazo mejorado se presenta con línea continua en color azul.

La figura 3 muestra una vista desde el acotamiento externo hacia la salida de la segunda curva en sentido hacia Puebla, durante la inspección en campo.

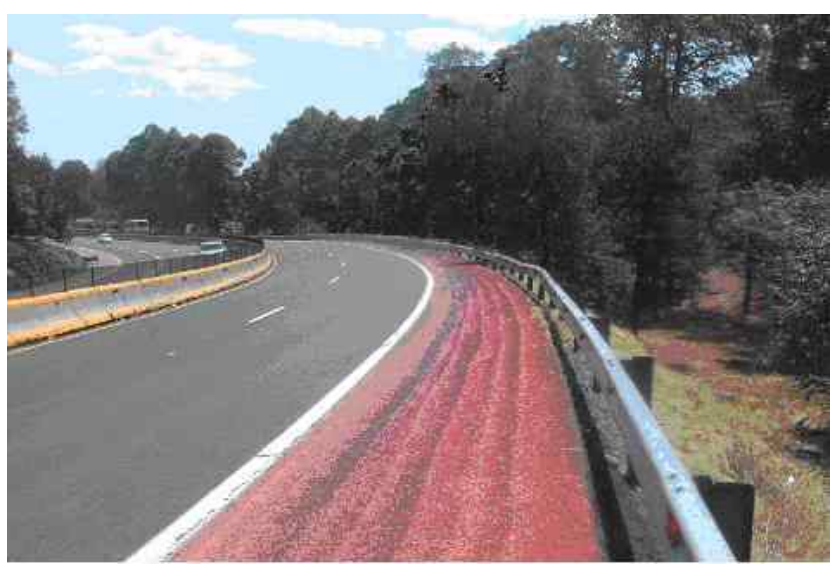

Figura 3. Fotografía de la segunda curva, durante la inspección de campo
La figura 4 presenta una vista de la misma curva, durante la realización de las obras de mejoramiento. La figura 5 ilustra el impacto de la auditoría, después de la realización de las mejoras.

\section{Beneficios y problemas}

En México, el incremento de la seguridad vial mediante el mejoramiento de la infraestructura se ha buscado a través de la atención de puntos negros o tramos peligrosos, que es un programa de tipo correctivo; así como de la realización de auditorías de seguridad de carreteras en operación, que es un programa de tipo preventivo. La inversión anual en mejoramiento de las carreteras (construcción, modernización y conservación) asciende al orden de $0.25 \%$ del Producto Interno Bruto.

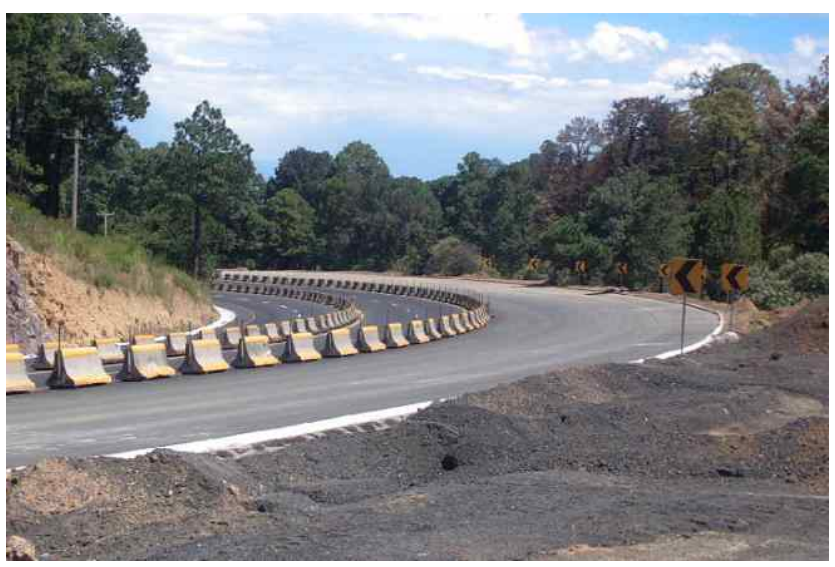

Figura 4. Fotografía de la segunda curva, durante la realización de los trabajos de mejoramiento

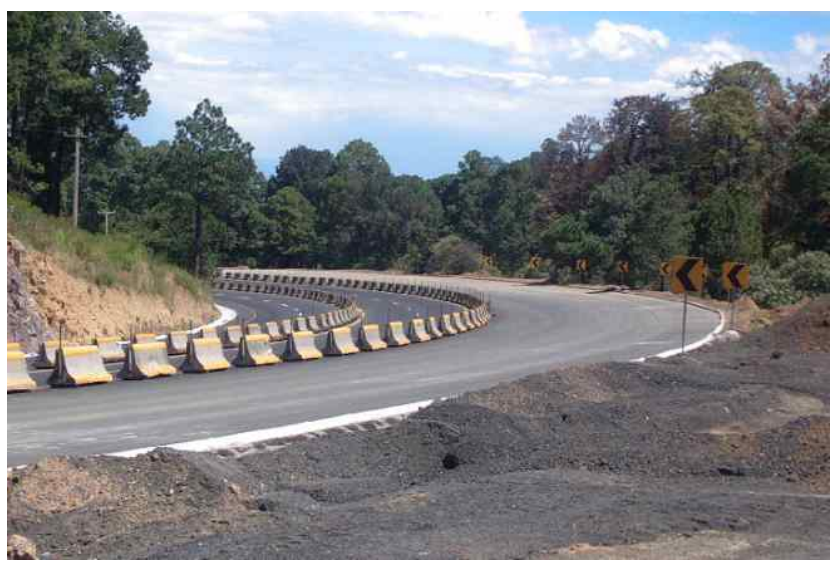

Figura 5. Fotografía de la segunda curva, con las recomendaciones de la auditoría 
Como resultado de estas medidas y de otras que se han venido realizando en el marco de un enfoque sistémico de administración de la seguridad vial, el índice de muertes por cada 100 millones de vehículos-kilómetro en la Red Carretera Federal se ha reducido de 6.5 a 4.4 en los últimos 10 años, a pesar de que los vehículos-kilómetro recorridos se incrementaron en un 33\% durante ese periodo.

Una carencia importante que existe en México es que no se cuenta con un documento regulatorio que norme la realización de auditorías. En algunos paises en los que esta normativa ya existe se establecen: los casos en los que la auditoría es obligatoria (en carreteras federales principales); sus etapas (factibilidad, proyecto preliminar, proyecto definitivo, construcción, preapertura y operación); el papel y responsabilidades de los diferentes actores (p. ej. organismo responsable de la carretera, organismo responsable del proyecto, equipo de proyecto, equipo auditor, etc.); el perfil requerido para los integrantes del equipo auditor; los procedimientos específicos para realizar la auditoría; etc.

Otro problema es que no existen auditores capacitados y certificados para realizar las auditorías. En la mayoría de los casos, las auditorías han sido efectuadas por ingenieros civiles con experiencia en proyectos, conservación y construcción de carreteras, pero sin mayor formación en ingeniería de seguridad vial. Será indispensable que se plantee como una exigencia al futuro la capacitación y certificación obligatoria de los equipos de auditores.

En México, según las disposiciones legales vigentes, primero deberán generarse organismos acreditados de certificación de auditores. Esta acreditación podrá otorgarla una asociación civil autorizada para ello por el Gobierno Federal, denominada Entidad Mexicana de Acreditación, AC (Secretaría de Comercio y Fomento
Industrial, 1991). Dentro de los organismos que pudiesen llegar a acreditarse como certificadores de auditores se encuentran los centros de investigación, las universidades, las asociaciones gremiales, etc.

En tanto no se cuente con los organismos nacionales acreditados para certificación de auditores, una alternativa la proporcionan algunas firmas internacionales de consultoría en seguridad vial, las cuales ofrecen cursos de certificación para ingenieros que deseen ser entrenados y certificados como auditores en seguridad vial. El costo por este servicio puede llegar a ascender a 20 ó 30 mil dólares por candidato.

\section{Agradecimientos}

Los autores agradecen al Instituto Mexicano del Transporte la oportunidad que les brindó de realizar el presente trabajo.

\section{Referencias}

Austroroads Road Safety Audits. Sydney, Australia. 2004. Pp. 100.

Secretaría de Comercio y Fomento Industrial. Autorización para Operar como Entidad Nacional de Acreditación a la Asociación Civil denominada Entidad Mexicana de Acreditación, AC (EMA). Diario Oficial de la Federación. México, DF. 1991.

SCT. Normas de Servicios Técnicos, Proyecto Geométrico: Carreteras. Dirección General de Servicios Técnicos. México, DF. 1984.

SCT. Reglamento sobre el Peso, Dimensiones y Capacidad de los Vehículos de Autotransporte que Transitan en los Caminos y Puentes de Jurisdicción Federal. Diario Oficial de la Federación. México, DF. 2000. 
DOI: http://dx.doi.org/10.22201/fi.25940732e.2009.10n2.013

Auditorías de seguridad vial de carreteras en operación

Semblanza de los autores

Alberto Mendoza-Díaz. Obtuvo el grado de ingeniero civil, con mención honorífica, en el Instituto Tecnológico y de Estudios Superiores de Monterrey (ITESM) en 1979. Asimismo, el grado de maestro en ciencias y de doctor en la Universidad de Texas en Austin en 1983 y 1986, respectivamente. Es coordinador de seguridad y operación del transporte del Instituto Mexicano del Transporte. Pertenece al Sistema Nacional de Investigadores (SNI) del Consejo Nacional de Ciencia y Tecnología (CONACYT). Es miembro activo de varias asociaciones gremiales relacionadas con la ingeniería civil, las vías terrestres y el transporte.

Emilio Abarca-Pérez. Es ingeniero civil egresado de la Universidad Autónoma de Chiapas en 2001. En 2003, obtuvo el grado de maestro en ingeniería con especialidad en vías terrestres de la Universidad Autónoma de Chihuahua. Es investigador de la Coordinación de Seguridad y Operación del Transporte del Instituto Mexicano del Transporte. Ha participado en diversos proyectos de investigación relacionados con la seguridad y operación del transporte.

Agustin Gerardo Centeno-Saad. Es ingeniero civil del Instituto Tecnológico de Durango, donde obtuvo el titulo en 2001. Cursó los estudios de maestría en ingeniería en 2003, con especialidad en vías terrestres en la Universidad Autónoma de Chihuahua. Es investigador asociado en la Coordinación de Seguridad y Operación del Transporte del Instituto Mexicano del Transporte, en donde desarrolla proyectos de investigación relacionados con la seguridad vial, sitios de alta incidencia de accidentes y modelación de redes de transporte. 